\title{
Commentary The risks may be too high
}

KENNETH R. SILK, University of Michigan Health System, Ann Arbor, MI, USA

\begin{abstract}
The proposed revisions to the diagnosis of personality disorders in ICD-11 move the diagnosis of personality disorders from the categorical to the dimensional. Although there may be a number of good reasons to consider changes in the manner in which we diagnose personality disorders, the method proposed here goes too far in the degree of changes that it proposes. It ignores the fact that at least for some of the personality disorders, there is data that supports them as distinct diagnostic entities separate from other axis I and axis II disorders. Eliminating the diagnostic categories that have been part of the established psychiatric nomenclature for the last 30 years threatens to undermine the significant research and clinical advances that have been made using categorical diagnoses. Copyright @ 2011 John Wiley $\mathcal{E}$ Sons, Ltd.
\end{abstract}

It appears that much thought and care went into the recent position paper by Tyrer et al., 'The rationale for the reclassification of personality disorder in the 11th revision of the International Classification of Diseases (ICD-11)', which appears in this issue of Personality and Mental Health. The paper is clearly written and makes a straightforward and cogent argument as to why we need a revision of the manner in which we go about (process) as well as the criteria that we use in arriving at a diagnosis within the personality disorder realm. The case presented here is somewhat different from that made by the DSM- 5 personality and personality disorders workgroup (APA, 2011; Gunderson, 2010; Skodol, 2011), although I suspect that there was more internal dissension in the DSM-5 group despite the fact that the paper here from the World Health Organization had authors from 10 different countries. The manuscript suggests that there were at least four areas that needed to be addressed in the process of reform of the current classification of personality disorders: '(a) The unsatisfactory current labels of personality disorder based on categories; (b) The wide variations in levels of personality disturbance and associated impairment that the current system includes; (c) The artificial dichotomy of a personality disorder diagnosis from no personality disorder and (d) the stigma attached to the diagnosis leading to its infrequent use'. This paper will attempt to touch on most of those issues, although most of the attention here will be paid to the issue of dimensional diagnosis.

Diagnosis involves both the process and the end result of knowing or perceiving (gnosis) how to separate things apart (dia) from one another. In current usage, we often think of diagnosis as the end result of that process (i.e. we arrive at a diagnosis), but in truth, diagnosis involves both the process by which we try to identify a disease by way of its signs and symptoms as well as the name or label finally given to the detailed description that discriminates the entity from others (W. B. Saunders Company, 1965). Kendell and Jablensky (2003) wrote that 'If the defining characteristic of the 
category is a syndrome, this syndrome must be demonstrated to be an entity, separated from neighboring syndromes and normality by a zone of rarity [italics added here]. Alternatively, if the category's defining characteristics are more fundamental - that is, if the category is defined by a physiological, anatomical, histological, chromosomal, or molecular abnormality - clear, qualitative differences must exist between these defining characteristics and those of other conditions with a similar syndrome' (p. 7).

The previous statement by Kendell and Jablensky would, in my opinion, suggest that in developing a diagnostic category, one should search for points of rarity that distinguishes it from other categories or that there should be clear qualitative differences present to help separate it from other conditions with similar clinical and/or laboratory presentations. Although the second sentence of the quote from Kendell and Jablensky may be in concert with the idea of dimensionality, it would seem that the first sentence with its emphasis on a zone of rarity would lobby against a dimensional approach to diagnosis. In a dimensional system, there is no zone of rarity. In a categorical system, there is a clear distinction between what is and what is not, and that would involve in some way having a zone of rarity that defines what is in the diagnostic category and what is not. A dimensional system does not achieve this but rather suggests that each increment or degree of severity or recruitment of an additional criterion pushes the process of arriving at a diagnosis a tiny bit closer to pathology or a little further away from the range of normality, whatever that might be. The broad classification of personality disorders based upon severity as proposed here would not meet that standard of true qualitative difference because each progressive classification along the personality-personality disorder continuum appears to be continuous with the class above as well as the class below with no clear criteria set or rating scale to determine when personality difficulty becomes personality disorder or when personality disorder becomes complex personality disorder and so on, save for the increased number of situations in which difficulties have arisen. Although Tyrer et al. suggest that categorical diagnoses should be eliminated because of criterion overlap, the scheme presented here contains much overlap without any zones of rarity. We are left here in the same boat that all clinicians attempting to make a psychiatric diagnosis are left in; that is, that psychopathology is very dependent upon the subjectivity of the clinical evaluator.

However, the five severity levels of personality disturbance as proposed may fit better with the second sentence of Kendell and Jablensky's statement in that there is a good attempt here to develop 'qualitative differences .... between [one condition's] defining characteristics and those of other conditions with a similar syndrome'. I agree with Tyrer et al. that the first step in the diagnosis of personality disorder needs to be deciding whether the individual we are assessing does or does not meet criteria for a personality disorder or at least whether there is a strong enough suspicion of the possible presence of a personality disorder that further time should be spent in evaluating the more specific nature of that personality disturbance. Both the ICD recommendations as described in the paper as well as the proposed DSM-5 (where the first question asked is 'Is impairment in personality functioning... present or not? (American Psychiatric Association, 2011)) involve a process of first determining whether there is the possibility of a personality disorder being present before proceeding further. Without this first step becoming a routine step, there will not be any progress made on clinicians failing to or simply ignoring the possible presence of a personality disorder. If this question related to the possible presence of a personality disorder is not raised initially, it will rarely be considered later although I am not sure that any change will get most harried primary care physicians to ask this question, at least in the United States.

But after establishing at least that there might be a personality disorder present and that one should attend to that issue, the system, in my opinion, fails because it stops providing relevant information; it suggests that all that we have learned about specific 
personality disorders in the past can be sacrificed to the concept of dimensionality. The argument is made that dimensional measures are used in other branches of medicines, and the example of blood pressure is often put forward. There are many more examples of this dimensional approach such as in diabetes, cardiac risk and anaemia. But within those dimensions, (1) there are clear cut-offs as to what defines pathology and (2) there are many disease entities that are clearly categorical such as metastatic disease, myocardial infarction and cerebral vascular accident (stroke) to name a few. Although one might have many risk factors say for a stroke, and those risk factors can be measured, for example, in how much of the lumen of a carotid or middle cerebral artery remains patent (determined by magnetic resonance imaging, CT scan or Doppler), the stroke does not occur until there are clinical symptoms present of a stroke. Once the stroke is determined, there may be severity measures that can help us evaluate how bad the stroke was, but nonetheless, it is a stroke and is clear and distinct from let us say, transient ischemic attacks, which could be viewed as a dimension of a potential stroke or a liability to have a stroke. But nonetheless, the category of stroke is not achieved until the signs and symptoms of the event and its sequelae are present (pregnancy is a state, if not a true diagnosis, where the diagnosis or condition is clearly categorical).

There are substantial differences in both training and approach to diagnosing mental disorders between psychology and psychiatry. Academic psychology has, as best as I can tell, most frequency applied a dimensional approach to issues of psychopathology and diagnosis of mental disorders. Psychology focuses on determining the continuum from normal (or nonpathological) to pathological. Medicine on the other hand, for good or for bad, deals with the pathological. Although it is true that medicine, at least in the United States, has been criticized for not doing enough with respect to prevention, in other words from working to help reduce the progression from normal or somewhat abnormal to true pathology or disease, nonetheless, medicine has studied and dealt with defining what is pathological and how one pathological state differs from another, perhaps in concert with Kendell and Jablensky's first sentence mentioned previously. Certainly there is neither a right nor a wrong to this dimensional/categorical argument. Rather it is a matter of approach, embedded in one's specific discipline, and as physicians, we deal with pathology and in distinguishing one pathological state from another and do not usually deal with how far a given patient is from normal. Once pathological, as determined most often through a categorical diagnosis, the situation demands treatment to make it less pathological. We would hope a return to normal as the ultimate outcome, but as we know from recent research, we are much better at getting people to remission (hopefully a sustained remission). But we much more rarely get them to full, sustained recovery (Gunderson et al., 2011; Trivedi \& Daly, 2008; Zanarini, Frankenburg, Reich, \& Fitzmaurice, 2010).

There are many aspects of the argument raised by Tyrer et al. that are valuable for further discussion, but space and the fact that others are also commenting on the paper lead me to restrain these comments. But a few merit some brief mention. The argument is made that the current categorical diagnoses lead to lost data. For example, a patient with four of nine criteria for borderline personality disorder is closer to 'pathology' (5/9 in DSM-IV-TR) than 'normalcy' and thus in a categorical system determining that there is no disease (i.e. no diagnosis on axis II because full criteria have not been met) on axis II fails to convey what is present. For this reason alone, it is suggested that we need dimensions. But one could just as easily establish the 'probable' condition of borderline personality dimension and then in parentheses write the number of criteria present. Not only would this allow a specific diagnostic category to be formulated, but also the number of criteria present would be a specific numerical measure of severity (Oldham \& Skodol, 2000).

Further, arguments have been made that there needs to be some way that we address the problem of stigma. I am surprised that the authors do not 
address the issue directly, that is, by saying that there is a substantial need to educate the public about what we have learned about the course and treatability of at least some personality disorders. When I was young, people never used the word cancer; it was a diagnosis that needed to be whispered rather than spoken out loud. And yet we now have cancer centres where people with cancer who know they have cancer go for treatment for their cancer, and this despite the fact that overall we still are not very good at effectively treating the cancer so that we may cure the patient (Kolata, 2009). If anything, the improvements come through early detection and more specific categorization through various types of genetic and other laboratory measures. But the stigma about cancer was overcome by educating people as to what it was and facing it head on rather than avoiding it or changing its name or developing a dimensional model (such as normal cells, somewhat atypical cells, greater atypical cells and cancer cells) because the prognosis was bad or because it meant for most people death. It is such education that we ought to put forth at least for borderline personality disorder, the category that we have the most empirical research data for, data not only about course and treatment but also about distinguishing it from other personality and affective disorders (Gunderson et al., 2011, Lequesne \& Hersh, 2004; Prossin, Love, Zubieta, \& Silk, 2010; Zanarini and Frankenburg, 2008; Zanarini et al., 1989).

Psychiatry is unique in that it attempts to understand psychiatric disorder not only in the current context and in the current social environment but also in its effort to understand and appreciate the illness and the person with the illness in terms of the person's individual history, early life experiences, genetic and environmental experiences and the opportunities, both genetic and environmental, to develop resilience. If we do not consider patients in their entire historical context, we fail to evaluate and appreciate fully how individuals get to where they are at the current moment, with the current symptoms and with the current psychological tools to deal with themselves, with others, with society and with their symptoms or disease (Tyrer, Sensky, \& Mitchard, 2003). In considering the process of the diagnosis of personality disorders, there is a history that needs attending to, an understanding of how the broader category of personality disorders has been understood over time. Newer dimensional schemes ignore that history since, by radically changing the approach to diagnosis, they interrupt the continuity with the past and suggest that if the old data fit we will take it and that if it does not fit we can simply dismiss it because there is no place in our current diagnostic system for it. Decades of thinking about and researching prodromal events, genetic predispositions, biological abnormalities, parental attitudes and others will be lost because the research is tied to specific diagnostic categories and not to prototypes or dimensions. Although I am sure that the proponents of a dimensional model of personality disorder do not mean to allow this to happen, it does raise the question of whether we are willing to risk 25-35 years of knowledge and research to put in place a model that represents a narrow point of view that has not in general been accepted by psychiatry or medicine rather than a system that builds on what we already know and attempts to move the field of psychiatric diagnosis forward in an incremental fashion.

\section{References}

American Psychiatric Association. (2011). Proposed revision. Personality disorders. http://www.dsm5.org/ProposedRevision/Ppages/Personality Disorders.aspx. Accessed 7 August 2011.

Gunderson, J. G. (2010). Revising the borderline diagnosis for DSM-V: An alternative proposal. Journal of Personality Disorders, 24, 694-708.

Gunderson, J. G., Stout, R. L., McGlashan, T. H. Shea, M. T., Morey, L. C., Grilo, C. M., Zanarini, M. C., Yen, S., Markowitz, J. C., Sanislow, C., Ansell, E., Pinto, A., \& Skodol, A. E. (2011). Ten-year course of borderline personality disorder. Psychopathology and function from the Collaborative Longitudinal Personality Disorders Study. Archives of General Psychiatry, 68, 827-837.

Kendell, R. E., \& Jablensky, A. (2003). Distinguishing between the validity and utility of psychiatric diagnoses. The American Journal of Psychiatry, 160, 4-12. 
Kolata, G. (2009). Advances elusive in the drive to cure cancer. The New York Times, 24 April, A1.

Lequesne, E. R., \& Hersh, R. G. (2004). Disclosure of a diagnosis of borderline personality disorder. Journal of Psychiatric Practice, 10, 170-176.

Oldham, J. M., \& Skodol, A. E. (2000). Charting the future of axis II. Journal of Personality Disorders, 14, 17-29.

Prossin, A. R., Love, T. M., Koeppe, R. A., Zubieta, J. K., \& Silk, K. R. (2010). Dysregulation of regional endogenous opioid function in borderline personality disorder. The American Journal of Psychiatry, 167, 923-933.

Skodol, A. E. (2011). Scientific issues in the revision of personality disorders for DSM-5. Personality and Mental Health, 5, 97-111.

Trivedi, M. H., \& Daly, E. (2008). Treatment strategies to improve and sustain remission in major depressive disorder. Dialogues in Clinical Neuroscience, 10, 377-384.

Tyrer, P., Sensky, T., \& Mitchard, S. (2003). Principles of nidotherapy in the treatment of persistent mental and personality disorders. Psychotherapy and Psychosomatics, 72, 350-356.

W. B. Saunders Company. (1965). Dorland's illustrated medical dictionary (24th ed.). Philadelphia: W. B. Saunders Company.
Zanarini, M. C., \& Frankenburg, F. R. (2008). A preliminary, randomized trial of psychoeducation for women with borderline personality disorder. Journal of Personality Disorders, 22, 284-290.

Zanarini, M. C., Frankenburg, F. R., Reich, D. B., \& Fitzmaurice, G. (2010). Time to attainment of recovery from borderline personality disorder and stability of recovery: A 10-year prospective follow-up study. The American Journal of Psychiatry, 167, 663-667.

Zanarini, M. C., Gunderson, J. G., Frankenburg, F. R., \& Chauncey, D. L. (1989). The revised diagnostic interview for borderlines: Discriminating BPD from other Axis II disorders. Journal of Personality Disorders, 3, $10-18$.

Address correspondence to: Kenneth R. Silk, MD, Professor of Psychiatry, University of Michigan Health System, Rachel Upjohn Building, PO Box 579, 4250 Plymouth Rd, Ann Arbor, MI 48109-2700, USA. Email: ksilk@umich.edu 